\title{
Brote Hospital San Rafael de Alajuela, primeros casos de SARS-Cov2 en Costa Rica
}

\author{
Marcela Leandro Ulloa, M.D, M.Sc ${ }^{1}$ \\ Gabriela Rodríguez Mora, M.Sc² \\ Andrea Arias Soto, M.Sc ${ }^{3}$ \\ Evelyn Seing Murillo, M.D ${ }^{4}$ \\ Enrique San Gil Caballero, M.D, M.Sc ${ }^{5}$ \\ 1. Caja Costarricense Seguro Social, Hospital Ciudad Neily, Costa Rica; Directora General; \\ mleandrou@ccss.sa.cr; https://orcid.org/0000-0003-4384-7503 \\ 2. Caja Costarricense Seguro Social, Hospital San Rafael de Alajuela, Costa Rica; \\ Enfermera Epidemiología grodriguem@ccss.sa.cr \\ 3. Caja Costarricense Seguro Social, Hospital San Rafael de Alajuela, Costa Rica; Enfermera \\ Epidemiología aariasso@ccss.sa.cr \\ 4. Caja Costarricense Seguro Social, Hospital San Rafael de Alajuela, Costa Rica; Médico \\ Epidemiología eseing@ccss.sa.cr \\ 5. Caja Costarricense Seguro Social, Hospital San Rafael de Alajuela, Costa Rica; Médico \\ Epidemiología esangil@ccss.sa.cr
}

\section{RESUMEN}

Objetivo. Describir el brote de SARS-Cov2, y las acciones realizadas para mitigarlo, ocurrido en el periodo del 24 de febrero al 28 de abril del 2020, entre los funcionarios, pacientes y cuidadores en un hospital periférico, en la ciudad de Alajuela, Costa Rica. Material y métodos. Se incluyó a todo funcionario, paciente y familiares que tuvieron contacto con caso índice positivo por PCR por COVID-19; además de los que presentaron síntomas compatibles con SARS-Cov2. Se les aplicó la ficha de investigación de caso sospechoso y probable de enfermedad respiratoria por 2019-nCov. Se tomaron dos muestras de aspirado nasofaríngeo. Resultados. De las 828 personas a quienes se les realizó la PCR, 37 fueron positivos. El brote inició en el Servicio de Ginecoobstetricia y Pediatría, todos en el II piso, todos contactos del caso índice. Conclusión. Este brote fue causado por caso índice como portados del virus SARS-Cov2, la causa del brote fue el contacto estrecho de los funcionarios y pacientes con personal positivo por COVID-19.

Palabras claves: brote, COVID-19, SARS-Cov2, Costa Rica, Hospital San Rafael.

\begin{abstract}
San Rafael de Alajuela Hospital outbreak, first SARS-Cov2 cases in Costa Rica Objective. Describe the SARS-Cov2 outbreak and the actions carried out for decreased it, it was occurred during the period from February 24th to April 28th, 2020, among health workers, patients, and caregivers in a peripheral hospital, in the city of Alajuela, Costa Rica. Material and methods. All health workers, patients and relatives who had contact with a positive index case by PCR for COVID-19, were included; in addition to those who presented symptoms compatible with COVID-19. The investigation form for a suspected and probable case of respiratory disease by 2019-nCov was applied to them. Two nasopharyngeal aspirate samples were taken. Results. 37 of the 828 people who underwent PCR were positive. The outbreak started in the Obstetrics and Gynecology Service. Conclusion. This outbreak was caused by index case as SARS-Cov2 virus carried, the cause of the outbreak was the close contact of health workers and patients with COVID-19 positive staff
\end{abstract}

Key words: outbreak, COVID-19, SARS-Cov2, Costa Rica, San Rafael Hospital.

\section{Introducción}

Los coronavirus son una extensa familia de virus que pueden causar enfermedades tanto en animales como en humanos. En los humanos, se conoce que causan enfermedades respiratorias que pueden ir desde un resfriado común, 
hasta enfermedades más severas como el síndrome respiratorio de Oriente Medio (MERS-CoV) y el síndrome respiratorio agudo severo (SARS-CoV)" (OMS, 2020a).

El coronavirus descubierto recientemente, que causa la enfermedad COVID-19, es un virus que no había sido identificado previamente en humanos. Existen, además, cuatro coronavirus humanos ( $\mathrm{HCoV})$ endémicos a nivel global: HCoV-229E, HCoV-NL63, HCoV-OC43 y HCoV-HKU1.

Como parte de la reseña de la evolución de la enfermedad en pandemia, se tienen los siguientes datos. El 31 de diciembre del 2019, el municipio de Wuhan, en Hubei, China, informó sobre un grupo de casos de neumonía con etiología desconocida. Un mes más tarde, con más de 9.700 casos en China y 106 en otros 19 países, el Director General de la Organización Mundial de la Salud (OMS) declaró este brote como "emergencia de salud pública de importancia internacional". El 11 de febrero, la enfermedad causada por el nuevo coronavirus fue denominada con el nombre COVID-19, abreviatura de "enfermedad por coronavirus 2019", por sus siglas en inglés. Finalmente, el 11 de marzo de ese año, la OMS declara pandemia a la COVID-19. (OMS, 2020a; OMS, 2020b; OMS, 2020c).

Los síntomas más frecuentes de la COVID-19 son la fiebre, la tos seca y el cansancio; además de otros, como cefalea, congestión nasal, mialgias y artralgias, conjuntivitis, dolor de garganta, diarrea y, en algunas ocasiones, anosmia (pérdida del olfato) y disgeusia (cambio en la percepción del gusto), así como erupciones cutáneas o cambios de color en dedos de manos o pies. Generalmente, estos síntomas son leves y de inicio gradual. Algunas personas infectadas solo presentan síntomas muy leves.

Cerca del $80 \%$ de individuos que adquieren la enfermedad se recuperan sin necesidad de hospitalización. Alrededor de 1 de cada 5 personas que contraen la COVID-19 presenta un cuadro grave y experimenta dificultades para respirar.

Los grupos con mayor riesgo a desarrollar complicaciones son los adultos mayores y aquellas personas con enfermedades preexistentes, como hipertensión arterial, problemas cardiacos o pulmonares, diabetes, cáncer, enfermedades inmunológicas, tratamientos que deprimen el sistema inmunológico como quimioterapias, esteroides, entre otros y tabaquistas crónicos. Niños y adolescentes tienen la misma probabilidad de infectarse que otros grupos de edades y pueden propagar la enfermedad. Sin embargo, las pruebas realizadas a la fecha sugieren que niños y adultos jóvenes tienen menos probabilidades de desarrollar una enfermedad grave, aunque esto no puede descartarse del todo; según lo indicado por la OMS en su página de actualizaciones en el 2020.

El Hospital San Rafael de Alajuela (HSRA), Caja Costarricense del Seguro Social (CCSS), es un nosocomio regional que cuenta con 280 camas censables. Posee servicios de emergencias, hospitalización y consulta externa. Cuenta, aproximadamente, con 1300 funcionarios distribuidos en los tres turnos que cubren las 24 horas del día. Este centro, fue el primer lugar donde se reportó a nivel país un brote por COVID-19.

\section{Materiales y métodos}

El periodo de estudio y análisis de los casos corresponde del 6 al 29 de marzo del 2020. Se tamizaron activamente los funcionarios y pacientes asintomáticos o sintomáticos que tuvieran contacto con personas positivas por COVID-19 dentro del hospital, en cualquier servicio.

La sintomatología que se buscaba fue fiebre y/o síntomas respiratorios y/o malestar general, que coincidieran con el tiempo de incubación, en relación con el tiempo de contacto con los funcionarios o pacientes positivos durante este periodo.

A todos los funcionarios, pacientes y familiares de funcionarios que se les realizó la prueba se les aplicó la ficha de Investigación de caso sospechoso y probable de Enfermedad respiratoria por 2019-nCov.

Se analizaron muestras de aspirado nasofaríngeo a 828 personas, en el periodo de estudio. Esto incluyó funcionarios, pacientes y familiares de funcionarios que tuvieron contacto cercano con pacientes sospechosos y confirmados por COVID19.

Las dos muestras de aspirado nasofaríngeo que se tomaron por paciente fueron procesadas y analizadas; una parte, en el Hospital San Rafael de Alajuela, y otras, en el Instituto Costarricense de Investigación y Enseñanza en Salud (INCIENSA), como laboratorio oficial del Ministerio de Salud (2017). Se realizaron pruebas de PCR para detección de COVID-19 aprobadas por la FDA.

Las muestras se mantuvieron refrigeradas $\left(4^{\circ} \mathrm{C}\right.$ a $\left.8{ }^{\circ} \mathrm{C}\right)$. Una de ellas se procesó en el centro de salud designado por la red de servicios públicos, buscando, como mínimo, el panel respiratorio indicado en el apartado de vigilancia de caso 
sospechoso del lineamiento nacional versión 6 (Ministerio de Salud y CCSS, 2020a).

La segunda muestra se custodió según las normas establecidas para su preservación en el laboratorio del centro de salud y se envió al INCIENSA cumpliendo los requerimientos establecidos para el transporte de muestras para detección de virus respiratorios, con triple embalaje y a $4{ }^{\circ} \mathrm{C}$ (Ministerio de Salud, 2018). El Centro Nacional de Referencia de Virología de Inciensa coordinó con el Centro Nacional de Enlace y la oficina local de Organización Panamericana de la Salud (OPS/OMS) para el envío de la muestra a un laboratorio de referencia internacional para confirmación, según la decisión del Grupo Técnico Nacional de Influenza de acuerdo con la situación epidemiológica y a las guías publicadas por la OMS/OPS (2020d), cumpliendo con lo establecido en las guías internacionales de transporte de sustancias infecciosas.

Ética, conflicto de intereses y declaración de financiamiento: los autores declaran haber cumplido con todos los requisitos éticos y legales pertinentes, tanto durante el estudio como en el manuscrito; que no hay conflictos de interés de ningún tipo, y que todas las fuentes financieras se detallan plena y claramente en la sección de agradecimientos. Asimismo, están de acuerdo con la versión editada final del documento. El respectivo documento legal firmado se encuentra en los archivos de la revista.

Ethical, conflict of interest and financial statements: the authors declare that they have fully complied with all pertinent ethical and legal requirements, both during the study and in the production of the manuscript; that there are no conflicts of interest of any kind; that all financial sources are fully and clearly stated in the acknowledgements section; and that they fully agree with the final edited version of the article. A signed document has been filed in the journal archives.

\section{Resultados}

En el gráfico 1 se muestra la cantidad de personal y pacientes sospechosos, confirmados y descartados durante el periodo del 6 de marzo al 29 de marzo del 2020.

\section{Gráfico 1. Número de sospechosos, confirmados y descartados por COVID-19, HSRA, marzo 2020}

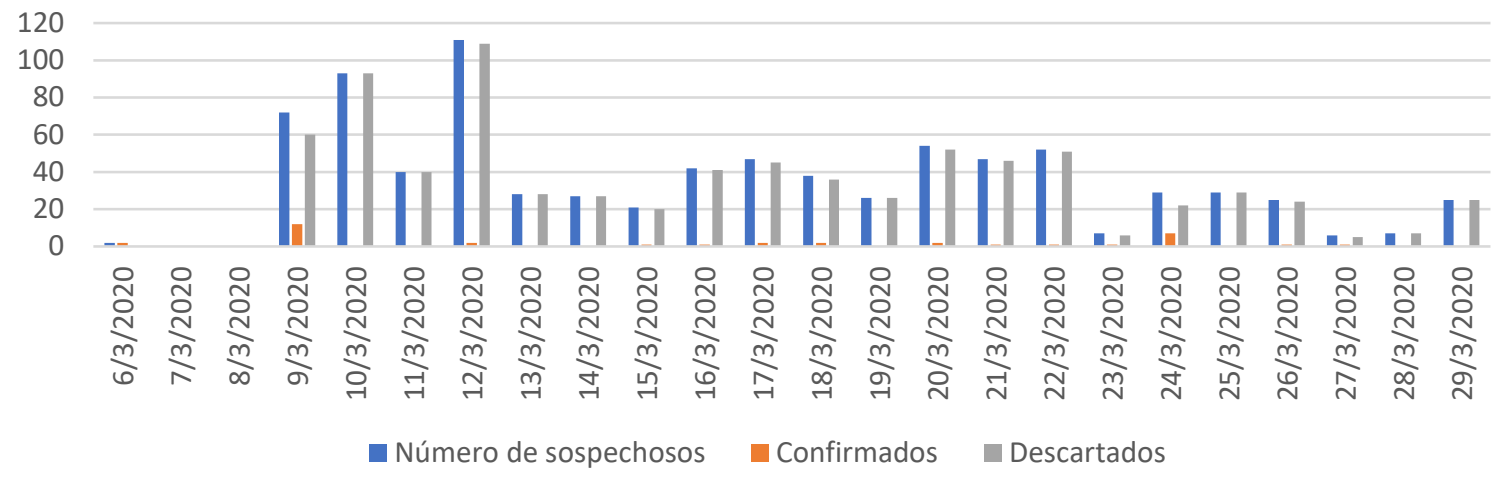

Fuente: Vigilancia Epidemiológica HSRA. Nota: No incluye al caso índice ni casos positivos diagnosticados en otros centros.

En total se realizó muestra de aspirado nasofaríngeo a 828 personas, entre funcionarios, pacientes y familiares de funcionarios que fueron contactos con personas sospechosas y confirmadas; de las cuales 36 salieron positivas por COVID-19 en la PCR realizada en el INCIENSA. Existe un funcionario positivo que fue diagnosticado en otro centro de salud, por lo que, en total, hubo 37 personas positivas en este brote.

Cuadro 1. Personas positivas por edad, sexo, fecha de inicio de síntomas y lugar de trabajo u hospitalización, HSRA, 2020

\begin{tabular}{|c|c|c|c|c|}
\hline Personas Positivas & Edad & Sexo & $\begin{array}{c}\text { Fecha de inicio de } \\
\text { síntomas }\end{array}$ & $\begin{array}{c}\text { Lugar de trabajo u } \\
\text { hospitalización }\end{array}$ \\
\hline
\end{tabular}




\begin{tabular}{|c|c|c|c|c|}
\hline AJR & 54 & $\mathrm{M}$ & $29 / 02 / 2020$ & II Piso \\
\hline PPS & 50 & $\mathrm{M}$ & $29 / 02 / 2020$ & II Piso \\
\hline GJR & 87 & $\mathrm{M}$ & $29 / 02 / 2020$ & Unidad COVID \\
\hline SPM & 58 & $\mathrm{~F}$ & $02 / 03 / 2020$ & II Piso \\
\hline MMF & 56 & $\mathrm{~F}$ & $03 / 03 / 2020$ & II Piso \\
\hline QAK & 38 & $\mathrm{~F}$ & $05 / 03 / 2020$ & II Piso \\
\hline MCM & 41 & $\mathrm{~F}$ & $05 / 03 / 2020$ & IV piso \\
\hline RVM & 73 & $\mathrm{~F}$ & $05 / 03 / 2020$ & IV Piso \\
\hline MSR & 50 & $\mathrm{~F}$ & $06 / 03 / 2020$ & II Piso \\
\hline PPM & 51 & $\mathrm{~F}$ & $06 / 03 / 2020$ & II Piso \\
\hline UOK & 45 & $\mathrm{M}$ & $06 / 03 / 2020$ & II Piso \\
\hline RSM & 37 & $\mathrm{~F}$ & $07 / 03 / 2020$ & II Piso \\
\hline GRJ & 43 & $\mathrm{M}$ & $09 / 03 / 2020$ & II Piso \\
\hline $\mathrm{OHC}$ & 87 & $\mathrm{M}$ & $09 / 03 / 2020$ & IV Piso \\
\hline VAK & 39 & $\mathrm{~F}$ & $09 / 03 / 2020$ & II Piso \\
\hline SLM & 39 & $\mathrm{~F}$ & $10 / 03 / 2020$ & II Piso \\
\hline RSM & 38 & $\mathrm{~F}$ & $12 / 03 / 2020$ & II Piso \\
\hline BS & 39 & $\mathrm{~F}$ & $12 / 03 / 2020$ & Unidad COVID \\
\hline PLM & 86 & $\mathrm{~F}$ & $12 / 03 / 2020$ & Unidad COVID \\
\hline GCI & 52 & $\mathrm{~F}$ & $12 / 03 / 2020$ & II Piso \\
\hline MVM & 42 & $\mathrm{M}$ & $12 / 03 / 2020$ & IV Piso \\
\hline HMM & 34 & $\mathrm{~F}$ & $13 / 03 / 2020$ & III Piso (arsenal) \\
\hline GGV & 32 & $\mathrm{~F}$ & $14 / 03 / 2020$ & IV Piso \\
\hline BCI & 43 & $\mathrm{~F}$ & $16 / 03 / 2020$ & IV Piso \\
\hline CMS & 21 & $\mathrm{~F}$ & $16 / 03 / 2020$ & II Piso \\
\hline MDG & 30 & $\mathrm{~F}$ & $17 / 03 / 2020$ & CGI \\
\hline SAJ & 33 & $\mathrm{M}$ & $18 / 03 / 2020$ & IV Piso \\
\hline JLR & 53 & $\mathrm{~F}$ & $19 / 03 / 2020$ & IV Piso \\
\hline SCD & 54 & $\mathrm{~F}$ & $19 / 03 / 2020$ & III Piso (arsenal) \\
\hline MMD & 31 & $\mathrm{M}$ & $21 / 03 / 2020$ & IV Piso \\
\hline AGO & 55 & $\mathrm{M}$ & $22 / 03 / 2020$ & III Piso (arsenal) \\
\hline GMG & 51 & $\mathrm{M}$ & $22 / 03 / 2020$ & Hospital Día \\
\hline MCT & 25 & $\mathrm{~F}$ & $23 / 03 / 2020$ & II Piso \\
\hline $\mathrm{OPC}$ & 40 & $\mathrm{~F}$ & $24 / 03 / 2020$ & IV Piso \\
\hline MRL & 39 & $\mathrm{~F}$ & $24 / 03 / 2020$ & IV Piso \\
\hline BAA & 51 & $\mathrm{~F}$ & $25 / 03 / 2020$ & US I Piso \\
\hline CVM & 26 & $\mathrm{~F}$ & $27 / 03 / 2020$ & IV Piso Unidad COVID \\
\hline $\mathrm{RCH}$ & 29 & $\mathrm{~F}$ & $03 / 04 / 2020$ & II Piso \\
\hline
\end{tabular}

Fuente: Vigilancia Epidemiológica HSRA. Nota: No incluye al caso índice ni casos positivos diagnosticados en otros centros. 
En el cuadro 2, se muestra la edad de los pacientes y funcionarios positivos, según sexo, fecha de inicio de síntomas y lugar de trabajo o de hospitalización. Según los datos recolectados en el cuadro, se nota que el brote inició en el servicio de Ginecoobstetricia y Pediatría, II Piso del Hospital, todos contactos del caso índice y de los casos secundarios al caso índice y se propagó por todo el hospital.

La mayoría de las personas positivas por COVID-19 durante este periodo son funcionarios del hospital (con un total de treinta y dos), tres pacientes hospitalizados y dos cuidadores de pacientes hospitalizados.

Es importante resaltar que todos los pacientes positivos tuvieron contacto estrecho con funcionarios o familiares positivos durante el periodo de incubación.

Se determinó que la edad promedio de las personas positivas en este brote fue de 46 años.

$\mathrm{Al}$ analizar la predominancia del sexo, se determinó que hubo predominio de sexo femenino (26) con respecto al masculino (11).

Se calcularon los coeficientes de infección hospitalaria en el brote, mismos que fueron:

a.-) tasa bruta de infección o tasa global de infección: 2.69\%,

b-) tasa específica de infección: $4.47 \%$ y

c.-) tasa de mortalidad por infección hospitalaria (letalidad): $5.4 \%$.

El caso índice, AJR (54) se diagnosticó el viernes 6 de marzo del 2020.

El total de pacientes que murieron en este periodo con PCR positiva por Cov-19 fueron dos, de 87 años cada uno.

\section{Discusión}

Las definiciones operativas de caso y contacto fueron tomadas de los Lineamientos Nacionales para la Vigilancia de la enfermedad COVID-19 Costa Rica, 28 de febrero 2020 (Ministerio de Salud y CCSS, 2020a), Versión Nº6.

Para este estudio se definió caso sospechoso como:

Todo trabajador del Hospital San Rafael de Alajuela y usuario que presente enfermedad respiratoria aguda de cualquier grado de severidad, que dentro de los 14 días anteriores al inicio de la enfermedad tuvo contacto físico cercano con un caso confirmado o probable por COVID-19. Donde se define contacto físico cercano, aquel contacto por más de 15 minutos a una distancia menor de 1.8 metros de distancia y sin protección, se incluyen aquí la realización de procedimientos que producen aerolización como intubación a pacientes sin protección adecuada.

En el periodo del 24 de febrero al 14 de abril del 2020, se presentaron en el Hospital San Rafael de Alajuela, 38 casos de personal y pacientes positivos por COVID-19, SARS-Cov-2, todos los casos de personal tuvieron nexo epidemiológico con algún funcionario del hospital, al igual que algunos de los pacientes internados en ese momento en el servicio de medicina. Es importante aclarar que 31 personas correspondieron a Infecciones asociadas a la atención de la salud, 6 familiares de funcionarios positivos y el caso índice.

En el cuadro 1 se nota que, en total, se les realizó la aspiración nasofaríngea a 828 personas, entre funcionarios, pacientes y familiares de funcionarios que fueron contactos con personas sospechosas y confirmadas, durante el periodo en estudio.

Un total de 36 personas fueron positivas por COVID-19, luego de la realización de la PCR en el INCIENSA. Es importante aclarar, que el 3 de abril del 2020 se diagnosticó un funcionario positivo, pero la toma de la muestra fue realizada en el Hospital San Francisco de Asís; por eso, no está incluido en el cuadro 1. En total, hubo 37 personas positivas en este brote.

Todos los pacientes positivos tuvieron un nexo epidemiológico con un paciente y/o funcionario positivo del centro.

$\mathrm{Al}$ analizar los periodos de incubación, se verifica que coinciden con el periodo del COVID-19.

El diagnóstico del caso índice ocurrió el 6 de marzo, pero inicia síntomas el 29 de febrero de 2020. El caso AJR, caso índice, salió del país y regresó de 22 de febrero de 2020. Posterior a esta fecha, retomó sus labores en el servicio de 
Ginecoobstetricia. El 23 de febrero de 2020 realizó atención de pacientes hospitalizadas, en emergencias y en consulta externa. Deja de trabajar el 4 de marzo del 2020, por presentar sintomatología.

El Hospital San Rafael de Alajuela tiene 4 pisos en donde se ubican las áreas de consulta externa, hospitalización y emergencias. En lo que respecta a las zonas de hospitalización, se distribuyen de la siguiente manera: en el segundo piso se encuentra Ginecoobstetricia, Sala de Partos y Pediatría; en el tercer piso, están sala de operaciones y todas las especialidades y subespecialidades quirúrgicas, junto con anestesia $\mathrm{y}$, en el cuarto piso, se encuentran todas las especialidades y subespecialidades de Medicina Interna.

El personal de enfermería se mantiene fijo durante la jornada ordinaria de trabajo en sus diferentes servicios; pero, en el tiempo extraordinario, pueden rotar a otros servicios. Lo mismo ocurre con el personal médico y especialistas, quienes, en el tiempo extraordinario, se trasladan desde hospitalización a emergencias o a sala de operaciones o de maternidad.

En el cuadro 2, se muestra la edad de los pacientes y funcionarios positivos, según sexo, fecha de inicio de síntomas y lugar de trabajo o de hospitalización. Según los datos recolectados en el cuadro, se nota que el brote inició en el servicio de Ginecoobstetricia y Pediatría, II Piso del Hospital, todos como contactos del caso índice y de los casos secundarios al caso índice. Por lo anterior, se decide realizar muestreo de todos los contactos de los funcionarios positivos del servicio de Ginecoobstetricia y Pediatría tanto de hospitalización, sala de operaciones como consulta externa.

En la semana del 8 de marzo al 16 de marzo de 2020, se tamizaron 434 personas y se diagnosticaron 17 funcionarios y pacientes. La mayoría de los funcionarios que se tamizaron eran de los servicios de Ginecoobstetricia y de Pediatría. Por lo anterior, se decide cerrar la consulta externa de todo el hospital, para limpieza de esta y disminuir la exposición de personas fuera del hospital.

De igual manera, se suspende el ingreso de pacientes a hospitalización de ambos servicios y se cierra la consulta externa por completo, además de los servicios de apoyo (RX, farmacia, laboratorio, REDES). Se prohíbe la entrada de visita de pacientes y, también, de estudiantes y personal de Organizaciones no Gubernamentales, para poder disminuir el contacto del personal del hospital con los usuarios y cortar la cadena de contagio.

En la semana del 17 marzo al 23 de marzo de 2020, se tamizaron 271 personas y se diagnosticaron 9 personas. En esta semana, se contó con funcionarios positivos que laboran en el IV piso Unidad de COVID, CGI, y SOP arsenal (III Piso), por lo que se inicia el cierre del IV piso. No se permite el internamiento de pacientes en medicina, ni cirugía, la sala de operaciones trabaja solo para emergencias calificadas y continúa la consulta externa cerrada completamente. Se toman muestras de los funcionarios y pacientes que tuvieron contacto con los funcionarios positivos.

En la semana del 24 de marzo al 28 de marzo de 2020, se tamizaron 121 personas y se diagnosticaron 9 personas positivas por COVID-19. En esta semana, se diagnosticaron trabajadores de sala de operaciones (III Piso), Aseo (II Piso), RX (I Piso), medicinas y unidad COVID. Por lo que se tamiza a las personas contactos estrechos de sospechosos y se incapacitan por 14 días. Se mantienen cerrados los servicios de hospitalización, sala de operaciones (solo para emergencias calificadas), consulta externa y servicios de apoyo.

Se mantiene en cuarentena las instalaciones del hospital en las cuales ha laborado personal positivo por COVID-19. Se mantiene un periodo de incubación para el cierre de estos servicios. El alta de la cuarentena del hospital se da el 16 de abril del 2020, que coincide con 23 días naturales posteriores al último caso positivo del personal del hospital.

Según el análisis de los resultados las fuentes de infección son los pacientes positivos con sintomatología respiratoria y gastrointestinal, que pueden ser infectantes desde 72 horas antes de inicio de síntomas.

El modo de transmisión es por gota y contacto y por aerolización (procedimientos realizados como intubación o RCP).

Debido a que la enfermedad por SARS-COV-2 es una enfermedad nueva y fue declarada pandemia por la Organización Mundial de la Salud en el 2020, cualquier cantidad de casos mayor de 0 se determina que es un brote. Por lo que, al tener en el periodo de estudio 37 casos positivos por COVID-19 en el Hospital San Rafael de Alajuela y que están relacionados directamente con el paciente índice, queda claro que sí se produjo un brote de SARS-COV2 en el nosocomio. Además, todos los positivos tuvieron contacto con el caso índice o con casos secundarios al índice.

El brote está condicionado por el hacinamiento de las personas en las estaciones de trabajo, en los comedores y por la mala higienización de las manos y la limpieza inadecuada de las superficies. 
Las medidas que sirvieron para poder cortar la cadena de transmisión de este brote fue el distanciamiento social, la segregación de casos confirmados y sospechosos en cohortes, el lavado de manos, las medidas de desinfección, el uso de equipo de protección personal y personal exclusivo para la atención de los salones de cohortes.

El aislamiento en cohorte se realizó de la siguiente manera: una cohorte con los pacientes diagnosticados y confirmados por COVID-19 mediante prueba de PCR de laboratorio positiva; una cohorte con los pacientes sospechosos con nexo epidemiológico fuerte con paciente positivo COVID-19 y una cohorte con los pacientes sospechosos, pero sin nexo epidemiológico.

En estos salones de pacientes solo pueden ingresar los pacientes que tengan como característica común ser positivo o sospechoso por COVID-19. No se pueden mezclar en un mismo salón de cohorte positivos y sospechosos. Además, se designa personal específico y exclusivo para la atención de estos pacientes.

Las acciones realizadas en vigilancia epidemiológica para atacar el brote fueron: listado y entrevista de todos los contactos de las personas positivas; toma de muestra para COVID-19 de los contactos estrechos de pacientes positivos que son sintomáticos; aislamiento de los contactos estrechos, sean sintomáticos o asintomáticos, por 14 días; seguimiento de las personas asintomáticas que inician síntomas antes de terminar los 14 días de aislamiento; capacitación del personal en uso adecuado de equipo de protección; generalidades sobre COVID-19; envío de personal de riesgo a teletrabajo u otras áreas que no tengan contactos con personal o pacientes sospechosos o confirmados; toma de muestra a toda persona con síntomas; todo personal que atiende a pacientes sospechosos y confirmados son exclusivos de esas áreas y se les da seguimiento diario a síntomas de cada funcionario; en las áreas de atención a pacientes sospechosos o confirmados, los funcionarios deben de utilizar ropa hospitalaria y dejar sus objetos personales fuera del área de atención; todo personal que atienda pacientes sospechosos y confirmados directamente debe utilizar equipos de protección; distanciamiento en las mesas del comedor y en el número de funcionarios sentados juntos por cada mesa; se marcaron zonas para la espera de pacientes en diferentes áreas del hospital, con distanciamiento de 1.8 metros; colocación de lavamanos en áreas de ingreso al hospital y a los salones de pacientes sospechosos o confirmados; separación física de espacios para la atención de pacientes sospechosos y confirmados (ejemplo cierre con puertas de los salones); colocación de solución alcohólica en áreas de atención a pacientes; atención a pacientes fuera de las instalaciones del hospital, en especial a las personas con riesgos; cierre técnico del nosocomio se realizó hasta que hubiese pasado un periodo de incubación después del último caso positivo reportado. Esa fecha fue el 14 de abril del 2020.

Algo importante que se debe aclarar es que este es el primer brote de SARS-Cov2 que se produce en Costa Rica. Son los primeros casos diagnosticados en el país y son las primeras acciones que se realizan para poder controlar la expansión de este. A partir de estas acciones es que se realizaron lineamientos nacionales para poder controlar futuros brotes (Ministerio de Salud y CCSS, 2020b).

\section{Agradecimiento}

Personal Asistencial y de Apoyo del Hospital San Rafael de Alajuela.

\section{Referencias}

Ministerio de Salud. (2017). Reglamento de Vigilancia de la Salud. DECRETO EJECUTIVO No 40556-S, Alcance digital No. 206 a la Gaceta del 23 de agosto de 2017. Recuperado de https://www.imprentanacional.go.cr/pub/2017/08/23/alca206_23_08_2017.pdf

Ministerio de Salud. (2018). Protocolo nacional para la vigilancia de personas con influenza y otras virosis respiratorias. (3 ed.). San José, Costa Rica: Grupo Técnico Nacional de Influenza. Recuperado de https://www.ministeriodesalud.go.cr/index.php/vigilancia-de-la-salud/normas-protocolos-yguias/inmunoprevenibles/3906-protocolo-nacional-para-la-vigilancia-de-personas-con-influenza-yotras-virosis-respiratorias/file

Ministerio de Salud y Caja Costarricense Seguro Social. (2020a). Lineamientos Nacionales para la Vigilancia de la Infección por Coronavirus 2019-nCoV, versión 6. Costa Rica: Ministerio de Salud.

Ministerio de Salud y Caja Costarricense Seguro Social. (2020b). Lineamientos Nacionales para la Vigilancia de la Infección por Coronavirus 2019-nCoV, versión 12. Costa Rica: Ministerio de Salud. 
Organización Mundial de la Salud. (2020a). Novel Coronavirus (nCoV) -Epidemiological Update v1. Recuperado

https://www.paho.org/hq/index.php?option=com content\&view=article\&id=15676:20-january2020-novel-coronavirus-ncov-epidemiological-update \&Itemid=42346\&lang=en

Organización Mundial de la Salud. (2020b). Coronavirus disease (COVID-19). Recuperado de https://www.who.int/health-topics/coronavirus\#tab=tab_1

Organización Mundial de la Salud. (2020c). Travel advice for international travel and health in relation to the outbreak of pneumonia caused by a new coronavirus in China. Recuperado de https://www.who.int/ith/2020-

0901_outbreak of Pneumonia_caused by a new_coronavirus_in_C/en/

Organización Mundial de la Salud. (2020d). Global Surveillance for human infection with novel coronavirus (2019-nCoV) Interim guidance. Recuperado de https://www.who.int/publications-detail/globalsurveillance-for-human-infection-with-novel-coronavirus-(2019-ncov) 